\title{
Lessons from International Experience with Electricity Market Monitoring
}

by

\author{
Frank A. Wolak ${ }^{1}$ \\ Department of Economics \\ Stanford University \\ Stanford, CA 94305-6072 \\ e-mail: wolak@zia.stanford.edu \\ http://www.stanford.edu/ wolak
}

\begin{abstract}
World Bank Policy Research Working Paper 3692, September 2005
The Policy Research Working Paper Series disseminates the findings of work in progress to encourage the exchange of ideas about development issues. An objective of the series is to get the findings out quickly, even if the presentations are less than fully polished. The papers carry the names of the authors and should be cited accordingly. The findings, interpretations, and conclusions expressed in this paper are entirely those of the authors. They do not necessarily represent the view of the World Bank, its Executive Directors, or the countries they represent. Policy Research Working Papers are available online at http://econ.worldbank.org.
\end{abstract}

\footnotetext{
${ }^{1}$ Professor of Economics and Chairman, Market Surveillance Committee, California Electricity Industry Independent System Operator. This paper was prepared as part of the research program on Industrial Organization Policy for Development at the Development Research Group of the World Bank, under the direction of Ioannis Kessides.
} 


\begin{abstract}
This paper first describes those features of the electricity supply industry that make a prospective market monitoring process essential to a well-functioning wholesale market. Some of these features are shared with the securities industry, although the technology of electricity production and delivery make a reliable transmission network a necessary condition for an efficient wholesale market. These features of the electricity supply industry also make antitrust or competition law alone an inadequate foundation for an electricity market monitoring process. This paper provides examples of both the successes and failures of market monitoring from several international markets. More than ten years of experience with the electricity industry re-structuring process has demonstrated that market failures are more likely and substantially more harmful to consumers than other market failures because of how electricity is produced and delivered and the crucial role it plays in the modern economy. Wholesale market meltdowns of varying magnitudes and durations have occurred in electricity markets around the world, and many of them could have been prevented if a prospective market monitoring process backed by the prevailing regulatory authority had been in place at the start of the market.
\end{abstract}




\section{Introduction}

There is a growing consensus around the world that a well-functioning wholesale electricity market requires a prospective market monitoring process. More than ten years of experience with the electricity industry re-structuring process has demonstrated that market failures are more likely and substantially more harmful to consumers than other market failures because of how electricity is produced and delivered and the crucial role it plays in the modern economy. Wholesale market meltdowns of varying magnitudes and durations have occurred in electricity markets around the world, and many of them could have been prevented if a prospective market monitoring process backed by the prevailing regulatory authority had been in place at the start of the market.

The purpose of this paper is first to describe those features of the electricity supply industry that make a prospective market monitoring process essential to a wellfunctioning wholesale market. Some of these features are shared with the securities industry. However, the technology of electricity production and delivery make a reliable transmission network a necessary condition for an efficient wholesale market. These features of the electricity supply industry also make antitrust or competition law alone an inadequate foundation for an electricity market monitoring process.

The next section draws lessons from wholesale electricity markets around the world for the design of an effective market monitoring process. The first lesson is that the market monitoring process should be forward-looking, anticipating how small market design flaws can develop into market failures that significantly harm market participants. The second lesson is that the market-monitoring process must be fully supported by the regulatory process. The regulator must have the willingness and ability to intervene to fix any problems discovered by the market monitoring process as rapidly as possible. The third lesson is the need for the market monitor to prepare consistent measures of market and system performance that are comparable over time and across markets. The fourth lesson is the need for public release of all data submitted to and produced by the market and system operators. Having this data readily available to all market participants will enable them to become more sophisticated players in the wholesale market in the sense of being better able to protect themselves against potentially harmful market outcomes. Public availability of this data will also allow other entities besides the market monitor and regulator to perform analyses of market performance. The final lesson is that the market monitoring process should be independent of the market operator, system operator and the political process. This will limit the incentive the market monitor might have to distort analyses of market or system performance to favor any stakeholder, instead of focusing on its role as an unbiased provider of analyses of market outcomes.

This paper provides examples of both the successes and failures of market monitoring from several international markets to illustrate these points. The United Kingdom (UK) provides an informative example of an electricity market that began operation with an inadequate market monitoring process. In fact, a major motivation for the New Electricity Trading Arrangements (NETA) was to implement a stronger market oversight process. New Zealand provides another example of the dangers of an inadequate market monitoring process. Its market was organized along the lines of the 
UK, but over the past three years it has experienced several sustained periods of extremely high prices that finally led to an overhaul of its market monitoring process. The United States (US) also had an inadequate market monitoring process at the start of its electricity re-structuring program. This allowed a number significant market performance problems to occur, including the highly publicized California disaster during the period June 2000 to June 2001, before the Federal Energy Regulatory Commission (FERC), the US wholesale market regulator, implemented significant reforms to its market monitoring processes.

There have been a few initial successes in electricity market monitoring. Australia provides perhaps the best example of the necessary conditions for an effective market monitoring process. There are also a number of positive lessons from the experience in the US, both before and after the reform of the electricity market monitoring process at FERC.

\section{Why Electricity Markets Are Different}

Electricity markets share many features with securities markets. Participants buy and sell rights to inject and withdraw electricity from the transmission network, much as securities investors buy and sell ownership shares of firms. Contrary to common perception, a buyer of electricity is not purchasing megawatt-hours (MWh) of energy produced by a specific generation unit. A buyer is only purchasing the right to withdraw that quantity of MWhs from a specific location in the network and a seller is paid for injecting a certain quantity of MWhs into the grid at a specified location in network.

Electricity markets differ from securities markets because virtually all electricity produced must be delivered to final consumers through the transmission network, and the actions of other market participants directly impact the ability of a market participant to sell or consume electricity. Consequently, all market participants share a common interest in reliable operation of the transmission network, even though they may often find it unilaterally optimal to engage in behavior that degrades overall grid reliability. For example, a supplier withholding capacity from the day-ahead market in order to increase the price it receives for the energy it sells, can create a reliability problem for all suppliers because the system operator is subsequently unable to dispatch the necessary generation units to the levels needed to meet real-time demand without increasing the risk of a system failure.

Two rationales for electricity market monitoring common to securities markets, are to ensure that: (1) both sides of each transaction comply with all contractual obligations and (2) maximum liquidity exists in the spot market. A necessary condition for an efficient wholesale electricity market is that participants produce or consume electricity under the terms and conditions specified in their bids into the market. Failure to comply with contractual obligations not only increases the cost to the violating firm of transacting in the future, it can also reduce the reliability of the transmission network and therefore the cost of other suppliers and load-serving entities transacting. A liquid spot market implies that unexpectedly large transactions can take place at virtually any instant in time without causing substantial price movements. If there is insufficient liquidity in 
the spot market, prices will be extremely sensitive to small changes in amount of energy purchased or sold in this market. An illiquid spot market can also reduce the liquidity of the forward market. If either side of the market is uncertain about the terms and conditions of delivery of a forward contract for electricity (including the spot price on the delivery date) or their ability to unwind this forward contract position at some future date, this will increase the cost of participating in the forward market and therefore reduce the liquidity of the forward market. These are the major reasons why electricity markets must be monitored to ensure there is sufficient liquidity in the spot and short-term forward markets.

In contrast to participants in financial markets, buyers and sellers of wholesale electricity have no choice about how to take or provide delivery on the electricity they have purchased or sold. All electricity must be delivered through the single transmission network serving a given geographic area. If the network is poorly operated, this increases the cost of participating in the wholesale electricity market for all entities. Inefficiencies in system operation not only impact prices in the spot market, but also the forward and futures market prices. The spot market is always an alternative option available to buyers and sellers, so it is the relevant opportunity cost of participating in the forward market. Consequently, there is a much greater need for market monitoring in electricity markets relative to financial markets because one supplier's privately profitable behavior can significantly degrade the ability of another supplier to inject the energy it has sold or for consumers at certain locations in the network to withdraw the energy they have purchased.

Because electricity markets require supply to equal demand at every instant in time at every location in the transmission network, and electrons flow according to the laws of physics rather than according to the terms of a financial contract, privatelyprofitable behavior by one market participant that degrades system reliability can immediately reduce the ability of other buyers and sellers to fulfill their contractual obligations. As noted earlier, because all suppliers deliver their electricity to final consumers through a common transmission network and if one supplier finds it profitable to inject significantly more or less energy into the network than the system operator expects, this can limit the ability of other suppliers and load-serving entities to inject and withdraw energy from the network. Market monitoring is therefore necessary to ensure that the unilateral profit-maximizing actions of certain market participants do not significantly degrade system reliability and market efficiency.

Electricity is also unique in the sense that the technology of producing and delivering electricity places significant constraints on how a wholesale electricity market can operate. For this reason, it is important to coordinate the design of the market protocols with the design of the engineering protocols used to operate the system. This fact implies that both engineering and economics expertise are essential to an effective market monitoring process, and these two sources of expertise must interact seamlessly.

Finally, this market monitoring process should be independent of the system and market operator and all market participants. One might think that this market monitoring 
function could be a department or division of the system operator. However, both market and system operation can often be undertaken at the expense of certain market participants. It is often the case that protocols that the system operator finds optimal can unnecessarily impose significant burdens on market participants. For this reason, it is essential to have an independent entity with no stake in market outcomes analyze and make recommendations on coordinating these two functions to ensure that system and market operating protocols enhance the efficiency of market outcomes, rather than simply make the system or market operator’s job easier.

The combination of the constraints on market operation caused by the engineering requirements to balance supply and demand second by second at all locations in the network, the high cost of storing electricity, the inelastic hourly wholesale demand (because of how electricity is priced to final consumers), and strict capacity constraints on production process make electricity markets extremely susceptible to the exercise of unilateral market power. Different from almost any other market, the exercise of unilateral market power can result in enormous wealth transfers from consumers to producers of electricity in very short periods of time. Because activities associated with the unilateral exercise of market power are not typically illegal under antitrust or competition law, these laws may be inadequate to prevent these wealth transfers. A market monitoring process is the most effective means of identifying and mitigating the harmful exercise of unilateral market power.

A final lesson from these episodes of significant unilateral market power from around the world is that it is extremely difficult to undo these wealth transfers by regulatory intervention after the fact. Consequently, a far more effective strategy is to prevent these events from occurring by vigilant market monitoring beyond that required by antitrust or competition law, with the goal of intervening before a small unilateral market power problem can become a large one and therefore result in enormous wealth transfers and potentially large efficiency losses.

\section{Lessons from around the World}

This section draws upon international experience with electricity industry restructuring over the past decade to illustrate the five major lessons described above.

\subsection{Need for a Prospective Market Monitoring Process}

Virtually all electricity markets around the world have experienced a sustained period with the exercise of significant unilateral market power. The markets that have fared the worst are those that did not have a prospective market monitoring process in place. The United Kingdom market is the earliest example, the California market in the US is the best-known example, and the New Zealand market the most recent example.

\subsubsection{United Kingdom Experience}

The electricity pool in the United Kingdom was formed in 1990 by a group of generation and distribution companies that jointly owned the National Grid Company (NGC), the operator of the transmission network and wholesale market. The specific role 
and responsibilities of the Pool was codified in the Pooling and Settlement Agreement (PSA), which all members signed. The Pool Rules specified the procedures which NGC used to operate the market, and gave the protocols for converting bids into prices. The PSA also contained a process for modifying the PSA, but the process turned out to be very convoluted.

This resistance to change was due to the fact that the Pool was governed by its members, with limited oversight by the regulatory body-the Office of Electricity Regulation (Offer) — or any other independent market monitoring entity. If a market design defect were identified, a sub-committee would be established to formulate a market rule change to solve the problem. This solution would then be voted on by the Pool Executive Committee (PEC). The PEC originally contained five generator representatives and five retailers. The structure was subsequently reformed to reflect the blurred distinction between generators and retailers (many companies were both) and allow consumer representation.

Because different market participants had voting weights based on their size, it was possible for the larger firms to block changes unfavorable to their financial interests. If a Pool member failed to obtain its desired outcome from participation in the Pool's own procedures, this dissenting company could still appeal to the regulator, further slowing the process of reform. A number of issues were deemed too contentious to resolve in time for the market opening in 1990, and were scheduled for subsequent resolution by the Pool governance process. Most of these created winners and losers, (which is why they could not be decided in 1990) and the Pool was never particularly successful in dealing with them.

The Office of Electricity Regulation (Offer) also found the non-responsiveness of the Pool governance process to problems in system and market operation increasingly difficult to deal with. For the reasons described above, Offer had little ability to implement prospective market rule changes to address small market design flaws before they caused significant harm. Moreover, the regulator was largely unable to implement market rule changes to address market design flaws that remained from the start of the market. For example, the problem of generation owner market power was identified early on and the need for divestiture of fossil-fuel generation capacity into a larger number of suppliers than National Power and PowerGen was recommended. However, ultimately the divestiture of some plant did not occur until the two dominant suppliers were subject to severe political pressure and substantial amounts of unilateral market power had been exercised for a number of years. In addition, the use of the capacity payment mechanism to enhance the ability of suppliers in the Pool to raise prices was identified during the early years of the operation of the Pool. Wolak and Patrick (1997) described this mechanism in detail and provided evidence of its use to raise market prices. Despite these inefficient market rules, they remained in place until the Pool was replaced by the New Trading Arrangements (NETA), which went into effect in March of 2001. Green (1999) provides a very informative discussion of the difficulty with implementing market rule changes and general regulatory oversight created by governance structure of the Pool. 
There were a number of highly publicized market rules changes implemented with NETA, but an important part of this reform process was to strengthen the governance process of the electricity market. The NETA market required all suppliers to sign a new license agreement - the connection and use of system (CUSC) agreementwhich gave Ofgem, the new combined gas and electricity regulator, the power to be a prospective market monitor with an enhanced ability to intervene to correct market design flaws. Specifically, the process of changing market rules in response to market design flaws has been significantly streamlined. One UK market commentator states with respect to the market rule change process that, "This is a great improvement on the stagnation that accompanied the change processes in the Pool, and it appears that Ofgem had delivered what it intended to-flexible governance.” (Cornwall, 2001). Moreover, different from the Pool regime, Ofgem now has the last word on all market rule changes. Both the flexibility in the change process and the ability to implement needed market changes are important components of the apparent success of NETA.

\subsubsection{The California Experience}

As discussed in detail in Wolak (2003a and 2003b), a major cause of the California crisis was the unwillingness of FERC and the California Public Utilities Commission to intervene to fix market design flaws identified by the Market Surveillance Committee (MSC) of the California Independent System Operator (CAISO). The MSC had identified the major market design flaws as early as of the Summer of 1998. The lack of incentives for the major California load-serving entities (LSEs) to engage in sufficient forward contracts for their retail energy obligations and the potential dangers associated with over-reliance on the spot market for their wholesale energy purchases were identified in the August 1998 MSC report to FERC (Wolak, Nordhaus, and Shapiro (1998)). This report also noted the need to face final consumers, particularly large industrial and commercial customers, with hourly wholesale price signals instead of a frozen retail price of electricity that was the same for all hours of the year. Although these warnings were repeated in subsequent MSC reports to FERC and a number of remedies were suggested, few of the necessary changes in the wholesale and retail market policies were implemented.

In addition, when the exercise of unilateral market power began to result in significant wealth transfers from consumers to electricity producers during the summer of 2000, FERC took no action to intervene to fix the market design flaws that created these circumstances. As discussed in Wolak (2003a), not until January 1, 2001 did FERC implement "remedies" for this exercise of unilateral market power. However, a number of independent entities, including both MSC and Market Monitoring Committee (MMC) of the California Power Exchange, argued that these remedies could very likely make matters worse. In fact, the market outcomes during the period January to June of 2001 are consistent with these "remedies" enhancing, rather than limiting, the ability of suppliers to exercise unilateral market power. In particular, after this implementation date, average spot prices rose to over $\$ 300 / \mathrm{MWh}$ and the first period of rolling blackouts 
immediately followed. As Wolak (2003a) notes, despite peak demands of over 44,000 MWh during the Summer of 2000 no rolling blackouts occurred. These first occurred following the implementation of the FERC "remedies" when peak daily demands were less than 33,000 MWh.

The purchase of substantial quantities of forward contracts by the California Department of Water Resources during the Winter and Spring of 2001 locked in a substantial quantity of energy for California during the Summer of 2001 and onwards. Conditions in the California market improved in mid-June 2001 when many of these contracts began making deliveries. However, attempts by California to obtain significant refunds from FERC for wholesale prices in excess of the "just and reasonable" levels required by the Federal Power Act of 1930 for the period June 2000 to June 2001 were largely unsuccessful despite an admission by the FERC that these prices were "unjust and unreasonable." In spite of FERC's unwillingness to order substantial refunds for these unjust and unreasonable prices, there has been a clear change in FERC policy in favor of prospective market monitoring over the past two years.

The Office of Market Oversight and Investigation (OMOI) at FERC was formed in early 2002. The OMOI has three major centers of activity-oversight/assessment, investigation/enforcement and planning/outreach. The first monitors market performance, the second follows any leads turned up by the monitoring process for further investigation to determine what regulatory actions should be taken, and the third provides strategy and interagency coordination. This new division of FERC currently employs more than 100 staff, with approximately half hired from other parts of FERC and the remainder hired from the outside. The recent downturn in the US energy sector has allowed the OMOI to hire a number of highly qualified candidates with significant industry experience, much to the benefit of the market monitoring process. Several members of OMOI are assigned to monitor each of the four currently operating wholesale markets in the US. In addition, some of the markets even have OMOI staff stationed at the site of the market operator to follow the day-to-day activities of the market and system operator.

In addition to a far more vigilant market monitoring process, FERC has also made a conscious policy change to become more forward-looking in its market oversight efforts by adopting policies designed to identify and address market design flaws before they can impose significant harm to market efficiency and system reliability.

\subsubsection{New Zealand}

New Zealand experienced a sustained period of extremely high wholesale prices during the period June to September of 2001 and again during the period June to September of 2003. In response, the New Zealand government abandoned its "lighthanded regulation" of the wholesale market. Prior to 2003, the New Zealand electricity industry was organized much the same way as the former UK electricity Pool. The industry was self-regulating and appointed an independent Market Surveillance Committee (NZMSC) to oversee its actions. 
Because it was appointed by the members of the industry and not backed by any formal government regulator, the NZMSC found it difficult to take positions contrary to the interests of the industry. For example, in response to the initial period of extremely high spot prices, claimed by two market participants to be due to the exercise of unilateral market power, the NZMSC issued a report investigating this "Claimed Undesirable Situation” in the New Zealand electricity market. Despite average prices during June 2001 that were more than 4 times the average prices for the previous year in a system with more than 65 percent hydroelectric capacity, the NZMSC concluded that, "the Committee does not find that an "Undesirable Situation" in NZEM (New Zealand Electricity Market) existed during 2001 (up to the end of June) and, in particular, in May and June of 2001." As a consequence, few actions were taken to prevent almost the same sequence of events during the same time period of 2003.

Partially in response to these events, the New Zealand Minister of Energy adopted the Electricity Governance Regulations in 2003, which established a seven-member Electricity Commission to take over governance functions. The Electricity Commission was also put in charge of taking actions to ensure that another period of extremely high prices would not occur in the future. The Electricity Commission also has the power to compel suppliers to offer long-term contracts for a pre-determined portion of their expected output. It is also responsible for modeling and forecasting future demand and supply conditions in the industry and determining transmission investment and pricing. In short, the Electricity Commission has given the New Zealand market a prospective market monitoring process with substantial powers to intervene in the market to prevent periods of sustained high spot prices such as those that occurred in June to September of 2001 and 2003.

\subsection{Need for Support from Relevant Regulatory Authority}

In virtually all cases, the primary reason wholesale market design flaws were not corrected before significant wealth transfers occurred was because of insufficient integration of the market monitoring process into the regulatory process. The US experience is most instructive in this regard because at the start of each regional wholesale market, FERC emphasized the importance of effective market monitoring protocols. However, FERC devoted little effort to designing a regulatory structure to enforce these market monitoring protocols. Because the UK Pool and New Zealand market were operated by market participants with little direct regulatory oversight, there was also little regulatory backing for the market monitoring process in these countries. With the possible exception of the Australian electricity industry, in all other countries of the world the failure to integrate the market monitoring process with the regulatory

process has been the major reason that market participants have failed to realize the full benefits of effective market monitoring. 


\subsubsection{United States}

Before a regional wholesale market could be approved, FERC required that the independent system operator (ISO) file a set of market monitoring protocols that would become part of the ISO's tariff. All ISOs were required to have internal market monitoring departments and several ISOs decided to include an independent market monitoring entity to oversee system operation and the operation of the wholesale market. These market monitoring protocols defined the duties of both the ISO's own market monitoring department and the independent market monitoring entity. However, there was very little specificity on how the ISO's internal market monitor and the independent market monitor would interact with FERC. When market design flaws were identified and called to the attention of FERC, an extremely cumbersome process was in place to attempt to correct these market design flaws. When the significant problem arose in the California market during the early Summer of 2000, FERC had no process in place to make use of the information provided by the California ISO's Department of Market Analysis (DMA) and Market Surveillance Committee (MSC) to implement market rule changes that would correct these market design flaws.

As is discussed above, FERC was well aware of the market design flaws in the California market long before the electricity crisis of June 2000 to June 2001 occurred. Moreover, throughout this period the DMA and MSC prepared numerous reports on the performance of the California market and made a number of recommendations for addressing the market design flaws they identified. These recommendations were largely ignored by FERC. Certainly one reason for this was the lack of any formal regulatory backing for the California market monitoring process by FERC. In particular, during this entire process, the analysis and comments of the DMA and MSC were treated in the same manner as those from other stakeholders, such as generation unit owners, load-serving entities and other state and federal entities. This occurred despite the fact that both market monitoring entities has access to confidential market outcome data (something none of the other stakeholders had) and prepared numerous reports on market performance that were submitted to FERC using this data. In short, the failure to integrate the market monitoring process into its regulatory process prevented FERC from benefiting from the analyses prepared by the DMA and MSC early enough in the process to implement the necessary market rule changes.

In fact, as noted above, because the OMOI division of FERC was not yet in place, the FERC staff (in Washington, D.C.) was not nearly as familiar with the workings of the California market as the DMA or MSC. The FERC staff report on the performance of the California market (that formed the basis for its "remedies" implemented in January 1, 2001) reflected this lack of familiarity with the market. It used only publicly available data and telephone conversations with market participants. Several parts of this analysis revealed significant misunderstandings of a number of aspects of the operation of the California market that could have easily been cleared up with more open lines of communication between FERC staff and the DMA and MSC. Consequently, it is perhaps not surprising that the "remedies" implemented as a result of this analysis led to more 
adverse conditions in the California market. Wolak (2003a) provides an analysis of this sequence of events.

Although the California crisis was largely solved by the decision of the State of California to purchase forward contracts during the Winter and Spring of 2001, a number of lessons have been learned by FERC from this experience. Specifically, it has attempted to increase the degree of coordination between FERC and the California market monitoring process. The most visible evidence is that two full-time FERC staff is stationed at the California ISO to interface with the ISO and FERC staff in Washington. In addition, FERC has relaxed its rules on ex parte communications during regulatory proceedings for market monitors. This has reduced the barriers to FERC staff interacting with the relevant market monitors at the various ISOs. The formation of the OMOI at FERC with specific staff devoted to each regional market is another step toward greater integration. Finally, FERC has also encouraged each of the ISOs to file oversight and investigation protocols that officially increase the degree of integration between the market monitoring process and FERC's regulatory oversight. These changes are very positive steps towards providing the necessary regulatory foundation for the market monitoring process, but this transition is far from complete.

\subsubsection{The United Kingdom}

The Pool regime in the UK had no independent market monitoring process besides that performed by Offer (the Office of Electricity Regulation). For the reasons discussed above, the major problem faced by Offer was its inability to implement the necessary market rule changes. The NETA has largely addressed this issue, although there still is a question of the adequacy of the market monitoring process for NETA.

A major issue of continuing debate in the UK is whether a Market Abuse License Condition (MALC) is a necessary part of the market monitoring process. During the formation of the NETA, Ofgem, the combined electricity and natural gas regulator, argued for a condition in the license or legal agreement that allowed each market participant to sell energy only if it agreed to be subject to penalties and sanctions for abusing its market power. The Competition Commission, the UK competition authority, was charged with determining whether such a provision should be included in the license for generation unit owner to sell power in the UK.

Ofgem wrote at the time, "Neither, we believe, can the potential abuse of substantial market power be adequately regulated under general competition legislation (although the Competition Act of 1998 provides a welcome strengthening of regulation against anti-competitive agreements and abuse of dominant positions) nor financial services legislation. This is because of particular physical and economic conditions associated with electricity wholesale markets and electricity networks--specifically the need for instantaneous (real-time) matching of supply and demand to maintain security and quality of supplies, coupled with the non-storability of electricity and the limited ability of the demand-side to respond to price movements in the very short-term.” (p. 2, Ofgem, 2000). 
Although a license provision prohibiting abuse of market power appeared to be an effective mechanism for limiting the exercise of market power, the difficulty Ofgem ran into was how to distinguish the unilateral exercise of market power from abuse of market power. It was never able to find a satisfactory way to make this distinction, and many economists including Wolak (2000), provided comments stating that this was not possible in a manner that did not introduce significant market inefficiencies. For this reason, Wolak (2000) argued against the implementation of a MALC. The Competition Commission eventually decided against a MALC, although periodically this issue reappears when prices in the UK begin to rise.

This debate about the need for a MALC raises a very important point about the goal of electricity market monitoring. Under US antitrust law and competition in law in the European Union, a finding that a firm has abused its market power requires an enormous amount of legal process and expense. The recent Microsoft trial in the US and European Union is a highly publicized example of such a process. The most difficult part of this process is making a finding of intent, because there are a number of benign explanations for market participant behavior that may appear to be an abuse of market power. Because of the need to follow legal process before making a determination of intent, the market monitoring process should instead focus on preventing significant harm from the exercise of market power, regardless of the reason for this harm. Consumers should be protected from significant harm that is the result of the legal unilateral actions of market participants, the unilateral abuse of market power, or coordinated actions among market participants to raise market prices. This does not mean that antitrust and competition authorities should not investigate firms for abuse of market power or collusive behavior, but because of the extreme difficulty in making such a determination, the market monitoring process should leave this decision to the relevant authorities and focus of preventing harmful market outcomes.

\subsubsection{New Zealand}

As noted above, until recently the New Zealand market had no formal regulatory commission. The Minister of Finance and Minister of Energy for the New Zealand Government oversaw the operation of the NZEM. Although there was an independent market monitoring committee, the NZMSC, its regulatory authority came from the agreement among members of the NZEM. This was in keeping with the strategy of "light-handed regulation" described above.

The NZMSC's July 17, 2001 report investigating an “Undesirable Situation” claimed by two NZEM market participants asserted that: “(1) excessively high prices are being set as a result of a lack of competition in NZEM, (2) offering and other activities of some generators may amount to manipulative activities under Rule 2.35.2, and (3) market conditions could represent conduct inconsistent with the high standards of integrity, fair dealing and of trading required by the Rules of NZEM” (NZMSC, p.1). This report did not analyze actual generator bidding behavior and was therefore unable to quantify the extent of unilateral market power exercised or the extent of system-wide market power 
reflected in the market-clearing prices during this period. The analysis was based primarily on water conditions in New Zealand, demand growth and the behavior of market prices. Consequently, it is not surprising the NZMSC was unable to reach any conclusions about the unilateral exercise of market power or the more serious charges of market manipulation.

The results of this investigation provide another example of the difficulty associated with making a positive finding of market manipulation. This experience, that in California and the discussion of the MALC in the UK underscores the necessity of shifting the focus of the market monitoring process way from a finding of market manipulation towards a finding of market conditions that impose significant harm to market efficiency and system reliability, regardless of their cause. If the unilateral profitmaximizing actions of market participants result in enormous wealth transfers from consumers of electricity to producers, then regulatory intervention should occur to fix the market design flaws that allow this to occur. The focus on a finding of market manipulation as a necessary condition for this to occur is misguided because of the virtual impossibility of a finding of market manipulation, without a finding of "intent" on the part of a specific market participant, as noted above. This underscores the need for a process to establish "intent to harm market efficiency and system reliability" as discussed in Wolak (2004). A necessary condition to make this determinant is a consistent set of measures of market performance, a topic discussed in the next section.

\subsubsection{Spain}

The Spanish market is another example of an electricity market with an inadequate market monitoring process. Although there is a formal regulatory commission for the electricity industry - Comision Nacional del Sistema Electrico (CNSE)--its efforts to implement market reforms that limit the ability of the two major suppliers in Spain to exercise unilateral market power have largely been undermined by the Spanish government. As early as 1999, CNSE was concerned with the lack of competition in the Spanish market and commissioned independent studies of the performance of the market. Unfortunately, these reports were never, to my knowledge, publicly released.

The studies concluded that both Endese and Iberdrola, the two major suppliers in Spain, had the ability to move market prices through their unilateral actions. However, the incentives of these firms to exploit this ability to raise prices were mitigated by a Competition Transition Charge (CTC) mechanism that still exists in the Spanish market. Several recommendations were made to reform this CTC mechanism to increase the competitiveness of the Spanish market. Unfortunately, the government did not follow these recommendations and current conditions in the Spanish market are still very similar to those that existed in 1999, with the incentives of Endesa and Iberdrola to raise wholesale prices mitigated only by the CTC mechanism. 


\subsection{The Need for Market Performance Measures}

Perhaps the greatest challenge faced by a market monitoring process is deciding when to intervene to fix market design flaws. Often there is substantial uncertainty about the underlying causes of a sustained period of potential harmful market outcomes. Without a clear understanding of the cause, intervention in the market runs the risk of making things worse rather than better. Intervention can also create incentives for behavior that will lead to harmful market outcomes in the future. If firms are confident that regulatory intervention will occur under certain market conditions they will be reluctant to incur the costs necessary to become more sophisticated market participants in the sense of being better able to formulate strategies that reduce the likelihood these harmful market conditions ultimately occur. For example, a low price cap on the spot market for electricity can significantly dull the incentive for retailers to sign forward contracts with generation unit owners which increases the likelihood of a unilateral market power problem in the spot market in the future. The commitment of the regulator to a low price cap also dulls the incentive for LSEs to sign forward contracts and make investments in technologies that allow price-response demand, which would eliminate the need for the price cap and improve the efficiency of the spot market.

Consequently, in deciding whether to intervene in the market, the regulator must balance these two costs of regulatory intervention against the expected benefits of the intervention. The costs of regulatory intervention can be reduced if the market monitoring process produces consistent and timely measures of market performance that the regulators can use to determine when intervention in the market is appropriate.

\subsubsection{United States}

The California electricity crisis is an instructive example of how not to intervene to correct market design flaws. As discussed above, FERC had little capability to diagnose market design flaws until long after June 2001. The OMOI division at FERC had not yet been created and FERC had not set up protocols to collect and analyze data from the various ISOs throughout the US. As emphasized in Wolak (2003a and 2003b), although FERC had a statutory mandate to set just and reasonable wholesale prices under the Federal Power Act of 1930, it had not yet defined a "just and reasonable price" for the wholesale market. This created considerable uncertainty in the California market during the Summer of 2000 regarding the likelihood FERC would intervene in the California market.

During the Summer of 2000, FERC Commissioners and staff made a number of public statements concerning FERC's intentions to ensure that wholesale prices in California were just and reasonable. As noted in Wolak (2003a), during the late Summer and early Autumn of 2000, many suppliers serving the California market expected that FERC would order refunds for the extremely high wholesale prices during the Summer of 2000. Evidence of this is the fact that the measure of market performance discussed in Borenstein, Bushnell and Wolak (2002) improved during the months of September and October of 2000 relative to the months of June and July of 2000. During early November of 2000, FERC issued its preliminary order on the "remedies" it proposed to implement 
in California. As discussed above, FERC's proposed remedies (that were eventually implemented) were generally acknowledged by a number of independent observers to enhance the ability of suppliers in California to raise wholesale prices. As a consequence, market performance in November and December 2000 was even worse relative to the previous two months. As noted earlier, after all of these remedies were fully implemented on January 1, 2001 the much-publicized rolling blackouts occurred and average spot prices rose to over \$300/MWh.

The likelihood that FERC implemented inappropriate remedies would have been significantly reduced if it had relied upon a consistent set of indexes of market performance to make its diagnosis of the flaws in the California market. In addition, had these indexes been monitored since the start of the California market, FERC could have timed its intervention to deal with the problems before they imposed significant harm on consumers. FERC currently requires the market monitors at all of the US ISOs to compile and report, at least on an annual basis, a set of market performance measures that can be compared across markets and over time. These indexes are designed to provide FERC with the necessary vital signs on market performance to diagnose design flaws before they can impose significant harm on market efficiency and system reliability. In addition, all of the market monitors are required to file with FERC an annual state of the market reports providing a comprehensive analysis of market performance.

\subsubsection{Other Markets}

The Australian market has a separate entity that prepares periodic reports on the performance of the National Electricity Market (NEM). The National Electricity Code Administrator (NECA) is charged with administering the National Electricity Code governing the operation of the NEM. Among NECA's objectives is "to collect information and statistics, publish reports and disseminate information relating to performance, administration, enforcement and adequacy of the National Electricity Code and the performance of the National Electricity Market.” (p. 4, NECA, 2001). Although the Australian Competition and Consumer Commission (ACCC) regulates the NEM, NECA oversees the operation of the NEM. NECA prepares weekly market analyses and quarterly statistical digests that monitor the performance of the market. These reports significantly enhance the effectiveness ACCC’s regulatory oversight.

In the UK, Ofgem currently serves the role of both the market monitor and regulator. It prepares annual reports on its regulation of the UK natural gas and electricity markets. In New Zealand the newly formed Electricity Commission will take over this monitoring and regulatory role. Spain continues to operate with CNSE as the market monitor and regulator.

\subsection{Public Data Release}

Public release of all data submitted to and produced by the system operator will increase the transparency of the wholesale market, particularly for the smaller players. Larger firms are more able to justify the expense of collecting the best available information on market outcomes. Consequently, rather than disadvantage these smaller 
firms, all data necessary to operate the spot market and the transmission network should be publicly released as soon as possible after the trading day.

An additional benefit of public data release is that all market participants are aware that their bids, schedules and output levels are publicly available. The fact that their behavior is directly observable is likely to make detection of market rule violations more straightforward. Any interested party can monitor the behavior of any market participant using this publicly available data.

Despite the many market performance advantages of public data release, few markets do so. The Australian NEM is one of the few markets that follows a data release policy of full disclosure the next trading day of all bids, schedules and output levels. FERC has adopted as very peculiar data release policy in the US. Bids, schedules and output levels are released with a six month lag, but market participant identities masked. This data release policy renders the resulting data significantly less useful for analysis of market performance and for providing sunshine regulation of the behavior of market participants. If a firm knows that its behavior is publicly observable, even with a six month lag, it will be less likely to engage in behavior that may cause public outcry. FERC's data release policy of concealing the identity of the market participant gives each market participant the ability to deny that they are engaging in the behavior found to exist for one or more firms. Each firm can always claim that they are not the ones engaging in this behavior or they are not the one engaging in the most objectionable form of this behavior.

Perhaps the best example of how public data release could have significantly enhanced the effectiveness of a regulatory process occurred during the California crisis. Because of data confidentiality restrictions it was impossible for the public, including the press, to analyze the bidding behavior of specific market participants. Although confidential reports on bidding behavior and market outcomes were submitted to FERC by the DMA throughout the crisis period, FERC was effectively able to ignore these analyses of bidding behavior because its data confidentiality requirements prevented these studies from being made available to other interested parties and the public. Had both the DMA and MSC studies, and the data they were based on, been released to the public immediately, it is difficult to see how the crisis would have lasted as long as it did. The public could have directly verified the levels of bids being submitted by all market participants and made their own assessment of the extent of the market power problem in the California market. The unwillingness of FERC to permit the release of the bid, schedule and settlement data from the California ISO prevented an open analysis and discussion of the causes of the California crisis. Instead, the crisis was allowed to continue because FERC did not take action based on the studies prepared by the DMA and MSC and it did not undertake its own analysis of the confidential data until the crisis period was largely over.

The UK Pool and New Zealand market had the policy of only releasing data to other members of the market. The UK Pool would allow other parties to purchase portions of the data at extremely high prices. The New Zealand market did not even 
allow the data to be purchased. Data release outside of the members of the NZEM was prohibited. This restrictive data release policy made it impossible to undertake independent analyses of the two price spike periods in June from September of 2001 and 2003. More recently, the New Zealand government has adopted a data release policy with a lag, similar to that in the US, although I am not aware of any researchers having gained access to this data.

\subsection{Independence of Market Monitoring Process}

The most effective market monitoring processes are those that are perceived by market participants as independent of the market and system operators and the political process. Public data release can be a commitment device for the regulatory process to maintain its independence. Under these conditions, any interested party has the same data as the regulator and can perform its own analysis of an issue raised by the regulator. By committing to immediate public data release, the regulator is giving all entities, including members of the press, the ability to perform their own analysis of the data and reached their own conclusions about the extent of market power being exercised and extent of harm being imposed on specific market participants. Therefore, full and immediate data release makes it extremely difficult for the regulator to conceal potentially harmful market outcomes to either side of the market from the public for a sustained period of time.

Market participants also face the risk that the regulator may make decisions that increase their costs or reduce their revenues. This regulatory risk increases the cost of participation in the wholesale market. To the extent that the market participants perceive the regulatory process as independent, this risk is reduced.

As noted above, operating the market or system to serve the needs of the market or system operator can often impose unnecessary costs on market participants. Consequently, a market monitoring process internal to the market or system operator may find it difficult to make recommendations for market rule changes that require greater effort by the market operator or system operator even if these changes best serve the needs of market participants. One role of the market monitoring process is to oversee the wholesale market operator and the system operator. For this reason, it must be independent of both of these entities.

\subsubsection{United States}

As California market participants discovered, once the political winds turned against the energy traders, due in part to the bankruptcy of Enron, a number of traders were arrested for actions that many observers argued had been going on since the start of the market in April of 1998 without objections from FERC and the California Public Utilities Commission. Although several of trading strategies outlined in the "Enron Memos” involved illegal activities, such as selling non-firm energy purchased outside of California as a firm energy delivery to California, a number of these strategies simply attempted to arbitrage differences in electricity prices across the markets over time and 
across locations. Nevertheless, once the Enron meltdown occurred, even these arbitrage strategies were used as evidence to convict Enron in the court of public opinion. A more independent regulatory process may have been able to distinguish between legal and illegal activities by the Enron traders. The actions by the state and federal criminal authorities in response to these memos have created significant regulatory risk for merchant energy suppliers. This has partially caused many of them to shut down or significantly downsize their energy trading affiliates. If one takes the position that many of the actions energy traders were arrested for in 2001 and 2002 routinely occurred in 1998 and 1999, then this sequence of events provides a valuable lesson about the importance of a regulatory process that is independent of the political process. If the regulatory process had been perceived as more independent, the Enron bankruptcy and prosecution of traders for illegal behavior could have occurred without the almost complete decimation of the energy trading sector.

\subsubsection{UK Experience}

The transition from the Pool to NETA in the UK was largely a political decision. Virtually all of the academics studying the UK Pool argued against this change. Their argument was that the divestitures of generation capacity and new entry that had taken place throughout the 1990s had largely solved the market power problem that existed during the early and mid-1990s. There was very little rigorous empirical analysis of the likely benefits of the reform. The high cost of obtaining the bids and market outcomes data made it difficult, if not impossible, for researchers to undertake such an analysis. Moreover, because Ofgem was a major advocate of NETA, it had little incentive to undertake such an analysis. The significant wholesale electricity price decline that occurred following the implementation of NETA has fueled a debate over whether it or the significant generation divestitures and new generation entry during the 1990's caused the price decline. Greater independence between the regulatory process and the political process could have allowed a more impartial and detailed analysis of the need to incur the significant cost associated with implementing NETA.

The UK Uplift Management Incentive Scheme (UMIS) implemented by Offer during the Pool regime provides an instructive example of the type of problems that might arise if the market monitoring function was part of the market or system operation. Under the Pool rules, National Grid Company (NGC) was responsible for managing the costs of all deviations from day-ahead energy schedules and procurement of the ancillary services necessary to run the system in real-time. These costs were recovered through a per MWh uplift payment. Over time these uplift costs rose substantially, until Offer implemented a regulatory mechanism that capped the maximum amount of uplift charges that NGC could pass on to consumers through the UMIS. As a result of the UMIS, the amount of uplift charges paid by consumers declined substantially. The experience of Offer with the UMIS underscores the significant market efficiency gains possible from effective monitoring of the performance of the system and market operator, as well as market participants. 


\subsubsection{Australia}

NECA fulfills many of the tasks of an independent market monitor for the Australian electricity market. It periodically prepares market analysis reports that are released to the market using data on bids, schedules and output levels released by the National Electricity Market Management Company (NEMMCO) on its web-site that day after the market operates. Although NECA's authority as the National Electricity Code administrator comes from the ACCC, NECA satisfies the independence criteria described above. Australia also has a number of other market monitoring entities. The Australian Bureau of Agricultural and Resource Economics (ABARE) is a government economic research agency noted for its independent research and analysis. It periodically prepares reports and analyses of Australian energy markets, including electricity. The National Competition Council was established by all Australian governments in November 1995 to act as a policy advisory body to oversee the implementation of National Competition Policy (NCP). It oversees competition policy as it is applied to the electricity industry. The Productivity Commission, the Australian Government's principal review and advisory body on microeconomic policy and regulation, conducts public inquiries and research into a broad range of economic and social issues including: competition policy, productivity, the environment, economic infrastructure, labor markets, trade and assistance, structural adjustment and microeconomic reform. It has also been an active participant in the Australian electricity reform process. The public availability of wholesale market data has allowed each of these entities to provide an informed perspective on conditions in the NEM to the ACCC and public at large.

\section{Conclusions}

One positive outcome from the California electricity crisis is that a market monitoring process is currently being put in place throughout the US to ensure that such a sequence of events will not occur again. Although there are still problems with interested parties gaining access to data from the wholesale market to perform the necessary analyses, FERC is currently working to put in place a market monitoring process that is forward-looking, fully integrated with and supported by its regulatory process, uses consistent measures of market performance and relies on independent market monitoring entities at the wholesale market level.

The Australian market provides an instructive example of the necessary foundation for a very effective market monitoring process: (1) rapid public disclosure of all relevant market data, and (2) the availability of entities with the time, expertise and legal mandate to prepare reports on market performance using this data. As I have emphasized previously, market monitoring is fundamentally about providing smart sunshine regulation (Wolak, 2004). Providing state-of-the-art analysis with the best possible data, and making this information available to the relevant regulatory authority is a necessary condition for effective market monitoring.

As the experience of the California ISO DMA and MSC during the period June 2000 to June 2001 illustrates, an additional necessary condition is that the regulatory 
authority fully supports the market monitoring process. This requires open lines of communication between the market monitor and the regulatory agency. The regulatory agency must also be willing to rely on the market monitor's analysis in regulatory decision-making.

A final point to emphasize from international experience is that market monitoring is a process of continuous improvement. All markets have had to modify their market monitoring protocols in response to events in their markets. The events in the US and New Zealand triggered enormous changes in their market monitoring process, but other countries have also implemented or are considering changes in their market monitoring processes to respond to events in their wholesale markets. 


\section{References}

Borenstein, Severin, Bushnell, James and Wolak, Frank (2002) “Measuring Market Inefficiencies in California's Restructured Wholesale Electricity Market, American Economic Review, December 2002, 1367-1405.

Cornwall, Nigel, (2001) "NETA-Is the Glass Half Empty or Half Full, September, available from http://www.ksg.harvard.edu/hepg/Papers/NCornwall\%20-\%20901.An\%20Initial\%20View.pdf.

Green, Richard (1999) "Draining the Pool: The Reform of Electricity Trading in England and Wales,” Energy Policy, volume 27, issue 9, pp. 515-525.

National Electricity Code Administrator (2001) "NECA Members Agreement," available from http://www.neca.com.au/.

Ofgem, (2000) "Introduction of a "Market Abuse" Condition in the Licenses of Certain Generators, available from http://www.ofgem.gov.uk/ofgem/whats-new/archive.jsp.

New Zealand Market Surveillance Committee, (2001), "Memorandum Re: Claimed "Undesirable Situation” arising from High Spot Prices in May/June 2001, available from http://www.m-co.co.nz.

Wolak, Frank, Nordhaus, Robert, and Shapiro, Carl, (1998) "Preliminary Report on the Ancillary Services Markets of the California Independent System Operator," August (available from http://www.caiso.com/docs/1998/12/18/1998121806493813577.pdf)

Wolak, Frank and Patrick, Robert H. (1997), “The Impact of Market Rules and Market Structure on the Price Determination Process in the England and Wales Electricity Market," available from http://www.stanford.edu/ wolak

Wolak, Frank (1999) "Market Design and Price Behavior in Restructured Electricity Markets: An International Comparison, in Competition Policy in the Asia Pacific Region, EASE Volume 8, Takatoshi Ito and Anne Krueger (editors) University of Chicago Press, 1999, 79-134.

Wolak, F.A. (2000) "Comments on the Office of Gas and Electricity Markets (Ofgem) License Condition Prohibiting Abuse of Substantial Market Power” July 31, 2000, available from http://www.stanford.edu/ wolak.

Wolak, Frank, (2003a), "Diagnosing the California Electricity Crisis," The Electricity Journal, August/September, 11-37.

Wolak, Frank (2003b) "Regulating Wholesale Electricity Markets in the Aftermath of the California Crisis,” The Electricity Journal, August/September 2003, 50-55. 
Wolak, Frank (2004), "Report on Monitoring Competition in the Central American Electricity Market” March, available from http://www.stanford.edu/ wolak. 